\section{Workforce Planning in UK Mountain Rescue}

Background.-The UK Mountain Rescue (MR) service, run entirely by volunteers, provides first response care for casualties and transports them to professional medical care. In the past decade the number of call-outs has increased, while national trends show a decline in volunteering. This decrease in volunteers could leave the MR service struggling to meet future demands.

Objective.-This paper examines reasons for the increase in the number of MR call-outs, rescue team methods for meeting the increased demand, and implications for volunteer management.

Methods.-An online survey was completed by 134 current members of UK MR teams; 383 outdoor enthusiasts who were potential MR volunteers completed an additional survey. Both surveys explored perceptions of the MR service and issues regarding workforce planning.

Results.-More than one third (36\%) of MR volunteers believed that the major cause of increased workload was supporting the statutory emergency services in nonmountain incidents. However, the majority of potential volunteers $(65 \%)$ believed that the major cause was increased participation in outdoor activities, and only $6 \%$ identified emergency service work as a cause. Current and potential MR volunteers were primarily motivated by the desire to assist fellow mountaineers and to help others in mountainous environments. In the future, recruitment might be improved by specific role descriptions, such as identifying less physically demanding support roles.

Conclusions.-The motivation of both current and potential MR volunteers is becoming increasingly incongruous with an increase in call-outs to assist emergency service support work in nonmountainous environments. That could lead to problems in both retention and recruitment. Specialized roles within the MR may address this issue to a degree, but increased use of volunteers to support public services may not be tenable. Further implications of this issue require further research.

Rohan Goel, BSc

Tom Nichols, BSc Will Jones, MBChB, FRCA Leeds, West Yorkshire, UK

\section{Field Warming of IV Fluids}

Background.-Several studies have been performed examining the use of heat packs from Meals Ready to Eat (MRE) to warm IV fluids. All have attempted to heat the fluid while it was still in the bag. In one study, that resulted in a delay of between 13 and 19 minutes in reaching the desired temperature. In a second study, there was a delay of 10 minutes, but only $500 \mathrm{~mL}$ of fluid was heated.
Objective.-The purpose of this study was to determine the feasibility of using MRE heat packs to adequately warm a bolus infusion of cold IV normal saline.

Methods.-The heat packs from 2 MREs were placed inside 1 plastic bag. The heat packs were activated with the recommended volume of water (time 0 ). Two sets of coils were made in 254-cm long IV tubing. Each set consisted of four 5-cm diameter coils held with duct tape. Both coils were placed between the activated heat packs in the plastic bag. A bolus infusion was simulated by running $5^{\circ} \mathrm{C}$ NS through the line with an $18 \mathrm{G}$ IV catheter. Fluid supply and infusion temperatures were recorded every second with thermocouples inside the fluid bag and inside the end of the IV tubing.

Results.-Ambient temperature was $3^{\circ} \mathrm{C}$. The IV fluid supply temperature was $5^{\circ} \mathrm{C}$ during the entire infusion. Simulated infusion temperature reached $35^{\circ} \mathrm{C}$ an average of 4.2 minutes after beginning setup. Average peak infusion temperature was $46.3^{\circ} \mathrm{C}$.

Conclusions. - This method of heating IV fluids with MRE heat packs can effectively raise infusion temperature to the recommended levels within 5 minutes. This method is independent of supply bag volume and reduces heat loss in transit as heating occurs in the tubing.

Aaron Hendrix North Bethesda, MD, USA

Mark A. Hendrix, MD Rockville, MD, USA

\section{The Novel Utility of Common Tools for Performing Field Amputation: What Is Safe and Easy to Use?}

Background.-Field amputations are sometimes required to extricate patients from the scene of an injury. It is not uncommon that traditional surgical equipment is neither available nor ideal, especially for cutting through bone. We evaluated the utility and safety of several nonsurgical tools readily available to most rescue units that could be used for field amputations.

Objective.-We sought to evaluate the difficulty and safety of using novel and nontraditional equipment in performing amputations.

Methods.-Our willed body program dedicated 24 cadavers for research. The 48 lower extremities were randomly assigned to varying operators and different tools to assess ease and time of amputation and presence of fracture. Several tools were compared: handheld multi-tools, a power saw, 2 types of tree loppers, a serrated knife, and hydraulic cutters. After amputation, the leg was disarticulated, inspected, and radiographed.

Results.-Of 48 legs, 21 (43.8\%) were amputated using a 24-inch Fiskar anvil tree lopper, 8 (15.7\%) using scissor loppers, $4(8.3 \%)$ using a Sawz-All, 2 (4.2\%) using a Gerber 06 knife and a Gerber 600 multi-tool saw, 5 (10.4\%) using a Leatherman Wave saw, and $8(16.7 \%)$ using the Jaws of Life. 